\title{
Micrurus snake venoms activate human complement system and generate anaphylatoxins
}

\author{
Gabriela D Tanaka', Giselle Pidde-Queiroz ${ }^{1}$, Maria de Fátima D Furtado², Carmen van den Berg ${ }^{3}$ and \\ Denise V Tambourgi ${ }^{1 *}$
}

\begin{abstract}
Background: The genus Micrurus, coral snakes (Serpentes, Elapidae), comprises more than 120 species and subspecies distributed from the south United States to the south of South America. Micrurus snake bites can cause death by muscle paralysis and further respiratory arrest within a few hours after envenomation. Clinical observations show mainly neurotoxic symptoms, although other biological activities have also been experimentally observed, including cardiotoxicity, hemolysis, edema and myotoxicity.

Results: In the present study we have investigated the action of venoms from seven species of snakes from the genus Micrurus on the complement system in in vitro studies. Several of the Micrurus species could consume the classical and/or the lectin pathways, but not the alternative pathway, and C3a, C4a and C5a were generated in sera treated with the venoms as result of this complement activation. Micrurus venoms were also able to directly cleave the $\alpha$ chain of the component C3, but not of the C4, which was inhibited by 1,10 Phenanthroline, suggesting the presence of a C3 $\alpha$ chain specific metalloprotease in Micrurus spp venoms. Furthermore, complement activation was in part associated with the cleavage of C1-Inhibitor by protease(s) present in the venoms, which disrupts complement activation control.
\end{abstract}

Conclusion: Micrurus venoms can activate the complement system, generating a significant amount of anaphylatoxins, which may assist due to their vasodilatory effects, to enhance the spreading of other venom components during the envenomation process.

\section{Background}

The Elapidae family is represented in America by three genera of coral snakes: Micruroides, Leptomicrurus and Micrurus, the latter being the most abundant and diverse group. In Brazil, M. corallinus and M. frontalis are responsible for the majority of coral snake envenomations. Although, Micrurus bites are relatively rare, the accidents can cause death, by muscle paralysis and respiratory arrest, few hours after envenomation [1].

The main feature of the coral snake action is the neurotoxicity, although, experimentally, it has been documented that some Micrurus venoms may produce myotoxicity and local lesions [2,3]. Furthermore, many enzymatic activities were detected in Micrurus venoms including phospholipase $\mathrm{A}_{2}$, hyaluronidase, phosphodiesterase, leucine amino

\footnotetext{
* Correspondence: dvtambourgi@butantan.gov.br

'Immunochemistry Laboratory, Butantan Institute, Av. Vital Brazil, 1500, São Paulo, 05503-900, Brazil

Full list of author information is available at the end of the article
}

peptidase, L-amino acid dehydrogenase and L-amino acid oxidase activities [4-6]. Only little or no proteolytic effects have been detected in Micrurus venoms [5,6].

Previously, we have analyzed the pro-inflammatory properties of the snake venoms from the Elapidae family, including the genera Micrurus (M. ibiboboca and M. spixii) and Naja (N. naja, N. melanoleuca and $N$. nigricollis), and demonstrated that these snake venoms can activate the complement system in normal serum [7]. The electrophoretic conversion of C3 was observed with all venoms in human normal serum containing normal concentrations of $\mathrm{Ca}^{2+}$ and $\mathrm{Mg}^{2+}$. However when $\mathrm{Ca}^{2+}$ was chelated the conversion of $\mathrm{C} 3$ was only observed in serum incubated with the $N$. naja and $N$. melanoleuca venoms. Purified human C3 was electrophoretically converted, in the absence of other complement system components, by the venoms from $N$. naja, $N$. nigricollis and $M$. ibiboboca. However, only the venoms from N. naja and $N$. melanoleuca contained a $144 \mathrm{kDa}$ protein
C Biomed Central

() 2012 Tanaka et al; licensee BioMed Central Ltd. This is an Open Access article distributed under the terms of the Creative Commons Attribution License (http://creativecommons.org/licenses/by/2.0), which permits unrestricted use, distribution, and reproduction in any medium, provided the original work is properly cited. 
recognized by anti-sera against cobra venom factor or human C3.

The complement system (C) consists of three activation pathways, i.e., classical (CP), alternative (AP) and lectin (LP) pathways, which converge at the proteolytic activation step of C3, the central component of the complement system. Complement activation may generate anaphylatoxins and membrane attack complex (MAC). Anaphylatoxins (C3a, C4a, and C5a) are considered the bridge between the innate and adaptive immunity, and they are responsible for control the local pro-inflammatory response through vasodilatation and the chemotaxis and activation of leukocytes [8-11]. The regulatory mechanisms of complement are extremely balanced, controlled by several complement inhibitors, such as membrane-bound complement regulators, and plasma proteins like C1-esterase inhibitor (C1-INH), which regulates activation of $\mathrm{C} 1$ and MASPs of the classical and lectin complement pathways, among others $[8,12,13]$.

The complement system not only plays an important role in the defense system, but also contributes to the amplification of inflammation if activated in excess or inappropriately controlled. The best-characterized venom that has a complement activating substance is that of the Cobra (Naja naja, Elapidae). Cobra venom contains a C3-like molecule named Cobra venom factor (CVF), which can initiate the alternative pathway by forming with factor B an extremely stable $\mathrm{C} 3 / \mathrm{C} 5$ convertase and the consequence of this is excess $\mathrm{C} 5 \mathrm{a}$ generation and $\mathrm{C}$ consumption [14-16]. We have recently investigated the complement activating properties of snake venoms of the Bothrops genus and found that several venoms caused complement activation [17]. The aim of the present study was to further investigate the action of snake venoms from the genus Micrurus, occurring in Brazil, on the activation pathways and components of the complement system.

\section{Results}

\section{Action of Micrurus spp venoms on the complement system activation pathways}

In order to evaluate the possible action of Micrurus spp venom toxins on the complement system, samples of normal human serum were incubated with PBS or the venoms and the remaining complement lytic activity was measured under conditions to develop the classical, alternative or lectin pathways. Figure $1 \mathrm{~A}$ shows that the venoms from $M$. ibibococa, $M$. altirostris, $M$ spixii and $M$. frontalis induced a significant reduction of the classical complement pathway lytic activity, as measured in haemolytic assays using antibody-sensitized sheep erythrocytes. Analysis of the activity of Micrurus spp venoms on the lectin pathway showed that $M$. ibiboboca, M. lemniscatus, M. altirostris, M. surinamensis and $M$. corallinus snake

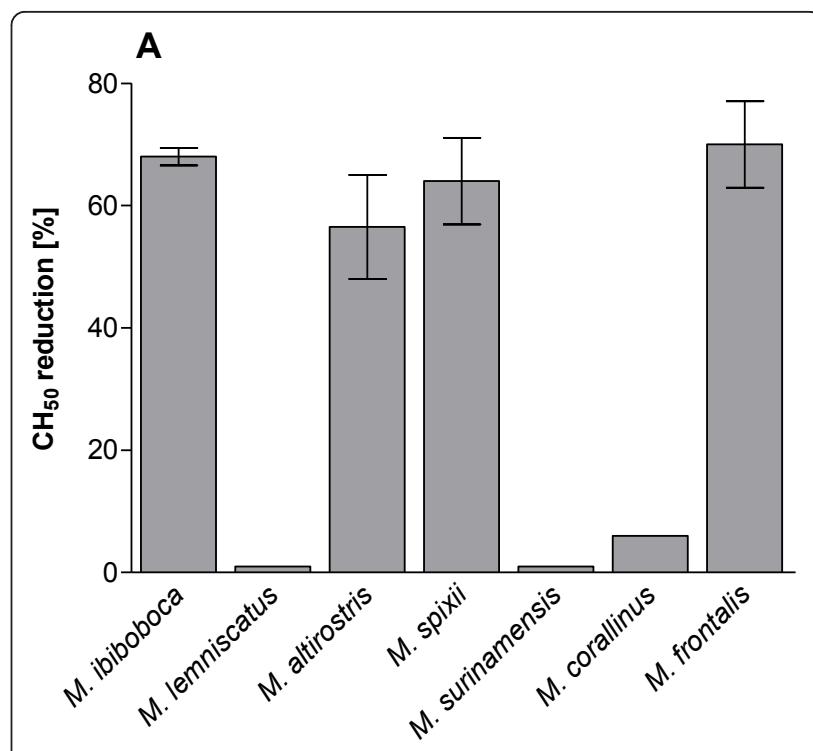

B

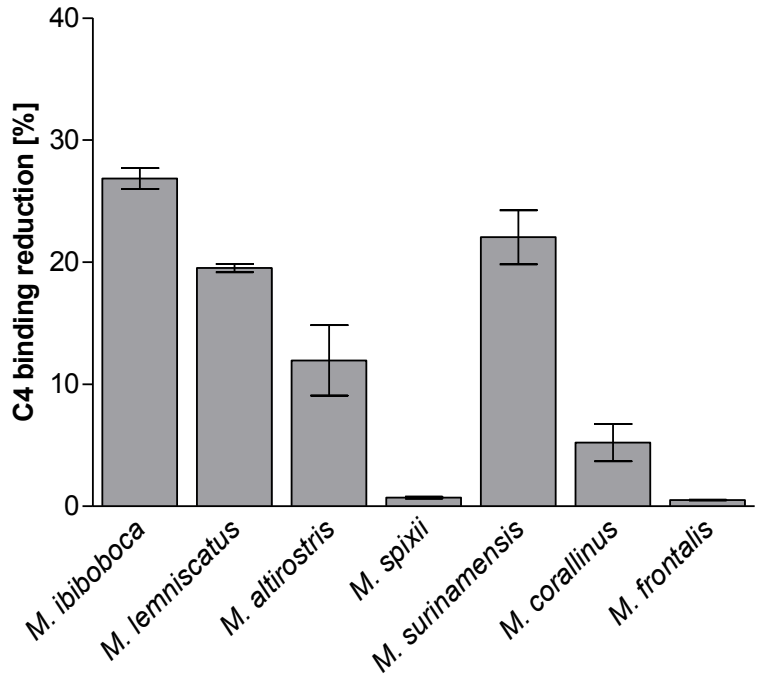

Figure 1 Action of Micrurus spp venoms on the complement pathways. Samples $(50 \mu \mathrm{l})$ of normal human serum (NHS), as complement source, were incubated with $50 \mu \mathrm{l}$ of Micrurus spp venoms $(50 \mu \mathrm{g})$ or PBS for $30 \mathrm{~min}$ at $37^{\circ} \mathrm{C}$. The residual complement lytic activity of the mixtures was measured using antibody-sensitized sheep erythrocytes, as targets, for classical pathway [A]. After incubation for $1 \mathrm{~h}$ at $37^{\circ} \mathrm{C}$, the absorbance of the supernatants was measured at $\lambda 414 \mathrm{~nm}$ and the percentage of haemolysis calculated. The residual lectin pathway complement activity [B] was determined on ELISA plates coated with mannan. Data are representative for three separate experiments and expressed as mean of duplicates +/-SD. Results were expressed as reduction of percentage of haemolysis [for $\mathrm{A}$ ] or reduction of $\mathrm{C} 4$ binding $[B]$ in relation to the control samples (NHS + buffer).

venoms induced a low but significant reduction of the LP activity (Figure 1B). No action was observed of Micrurus spp venoms on the alternative pathway (data not shown). 
To investigate if the decrease in the pathways activities, induced by Micrurus venoms, could be due to the presence of Cobra Venom Factor (CVF) like components, venoms were analyzed by western blotting using an antiserum raised against CVF from $N$. naja. Figure 2 shows that CVF components were clearly detected in N. naja and $N$. melanoleuca venoms. On the other hand, no CVF antigenically related components were detected in N. nigricollis, N. mossambica or Micrurus spp venoms.

\section{Induction of the anaphylatoxins by Micrurus spp venoms}

To assess if the above observed reduction in complement activities was caused by $\mathrm{C}$-inhibition or $\mathrm{C}$-activation/consumption, the generation of $\mathrm{C}$-activation products $\mathrm{C} 3 \mathrm{a}, \mathrm{C} 4 \mathrm{a}$ and $\mathrm{C} 5 \mathrm{a}$ was measured. Figure 3 shows that the majority of Micrurus spp venoms induced the production of significant amounts of the three anaphylatoxins. $M$. corallinus venom did not induce C5a release, but considerable amounts of C3a and $\mathrm{C} 4 \mathrm{a}$. These data suggest that the reduction in $\mathrm{CP}$ and LP, as observed above, is due to $\mathrm{C}$-activation/consumption rather that $\mathrm{C}$-inhibition.

\section{Micrurus spp venoms cleave the $\mathrm{C} 3 \alpha$ chain}

In order to analyze the possibility of direct proteolytic action of Micrurus venoms on the complement molecules, purified samples of $\mathrm{C} 3$ were incubated with the venoms and analyzed by western blot for the presence of cleavage fragments. Figure 4A shows that the venoms from $M$. ibiboboca, $M$. lemniscatus, $M$. corallinus, $M$. frontalis were able to induce cleavage of the $\mathrm{C} 3 \alpha$ chain $\left(\mathrm{M}_{\mathrm{r}} \sim 115 \mathrm{kDa}\right)$, but not of the $\beta$ chain, generating a fragment with an

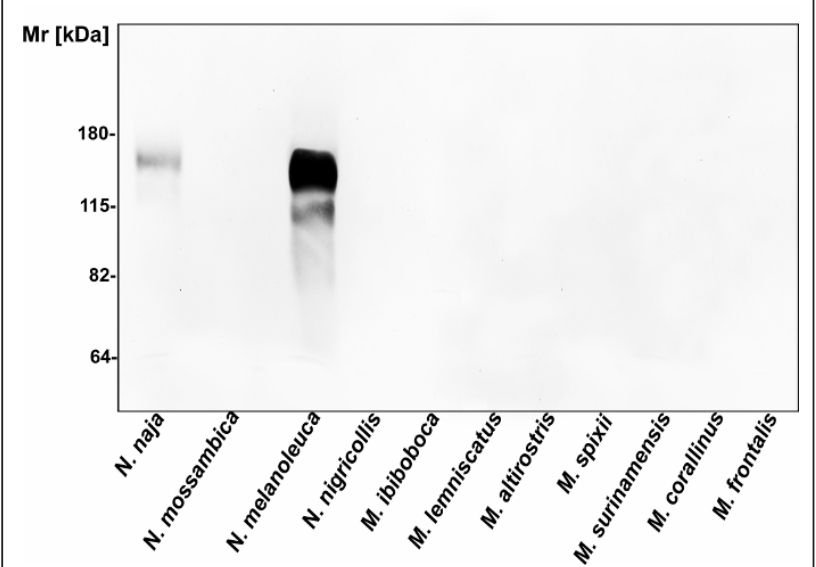

Figure 2 Analysis of the presence of CVF components in Elapidae snake venoms. Samples of $10 \mu \mathrm{g}$ of the Micrurus spp and Naja spp venoms were solubilised in non-reducing sample buffer and run on 10\% SDS-PAGE gel. The presence of CVF components was evaluated by Western blot using anti-CVF rabbit antiserum, diluted 1:1500.

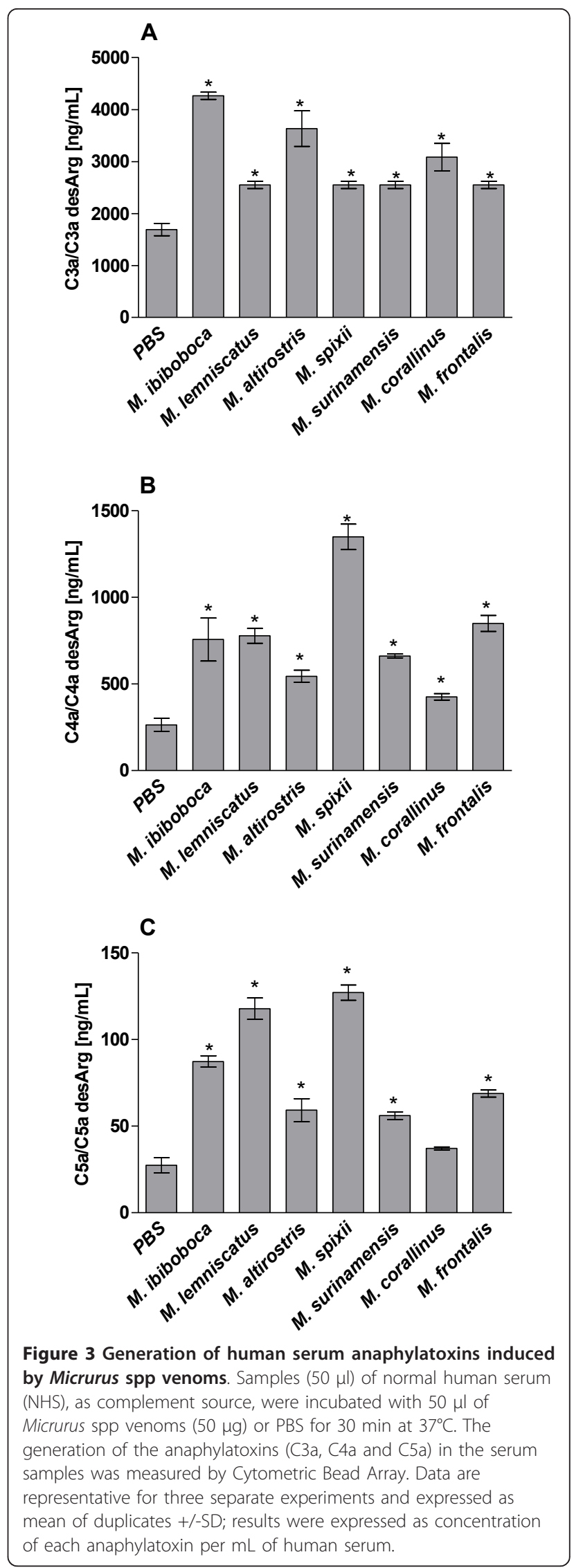




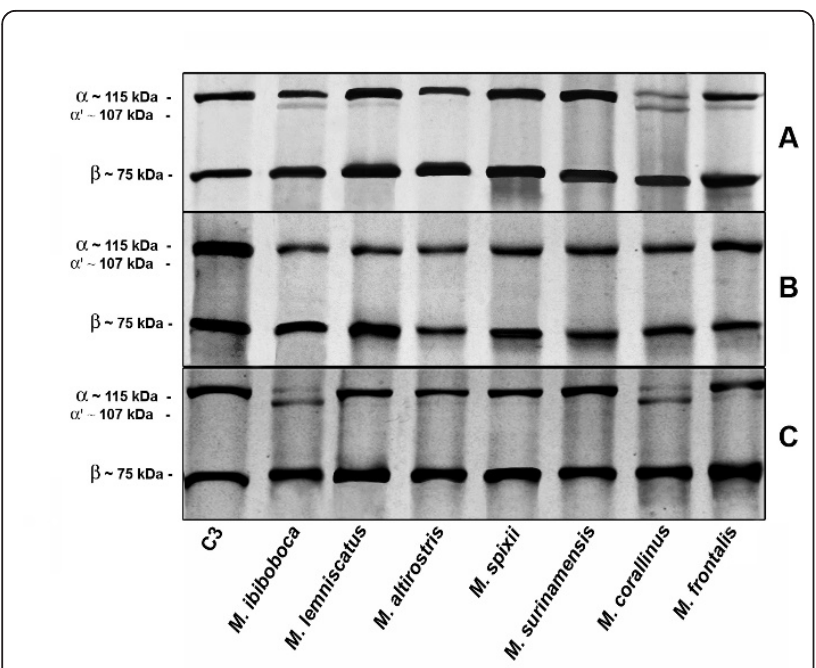

Figure 4 Proteolytic action of the Micrurus spp venoms on human purified C3. [A] Samples of purified component C3 $(1 \mu \mathrm{g})$ were incubated with $2 \mu \mathrm{g}$ of Micrurus spp venoms or PBS for 30 min at $37^{\circ} \mathrm{C}$. Inhibition assays were performed by including $10 \mathrm{mM}$ of 1,10 phenanthroline $[\mathbf{B}]$ or PMSF $[\mathbf{C}]$ in the mixtures. Cleavage was visualized by western blot using anti-C3 serum.

estimated $\mathrm{M}_{\mathrm{r}}$ of $107 \mathrm{kDa}$. The $\mathrm{C} 3 \alpha$ chain cleavage, induced by the venoms, could be prevented by the use of 1,10 phenanthroline, a metalloproteinase inhibitor (Figure 4B), but not by PMSF, a serineprotease inhibitor (Figure 4C). Analysis of the Micrurus venoms action on purified human $\mathrm{C} 4$ showed that all venoms were unable to induce cleavage of the $\alpha, \beta$ or $\gamma$ chains (data not shown).

Micrurus spp venoms induce cleavage of the C1-Inhibitor Since the majority of Micurus venoms could interfere with the classical and/or lectin pathways, we have investigated if the complement regulator of activation of these pathways, C1-INH, would have undergone to cleavage. Figure $5 \mathrm{~A}$ shows that all venoms induced cleavage of C1-INH, generating a main fragment of approximately $83 \mathrm{kDa}$. The fragmentation could be totally blocked by incubating venoms and C1-INH samples in the presence of 1,10 phenanthroline; PMSF could only partially abolish the cleavage of C1-INH induced by M. ibiboboca venom (Figure 5B and 5C).

\section{Discussion}

The toxins present in venoms, used by snakes for effective immobilization of prey and for protection against predators, must rapidly reach the target organs. Therefore, mechanisms facilitating the distribution of the toxins throughout the tissues of the bitten animal must be activated. Hyaluronidase, which is present in almost all venoms, was the first of such mechanisms to be described [18]. Another mechanism which could fulfill this spreading

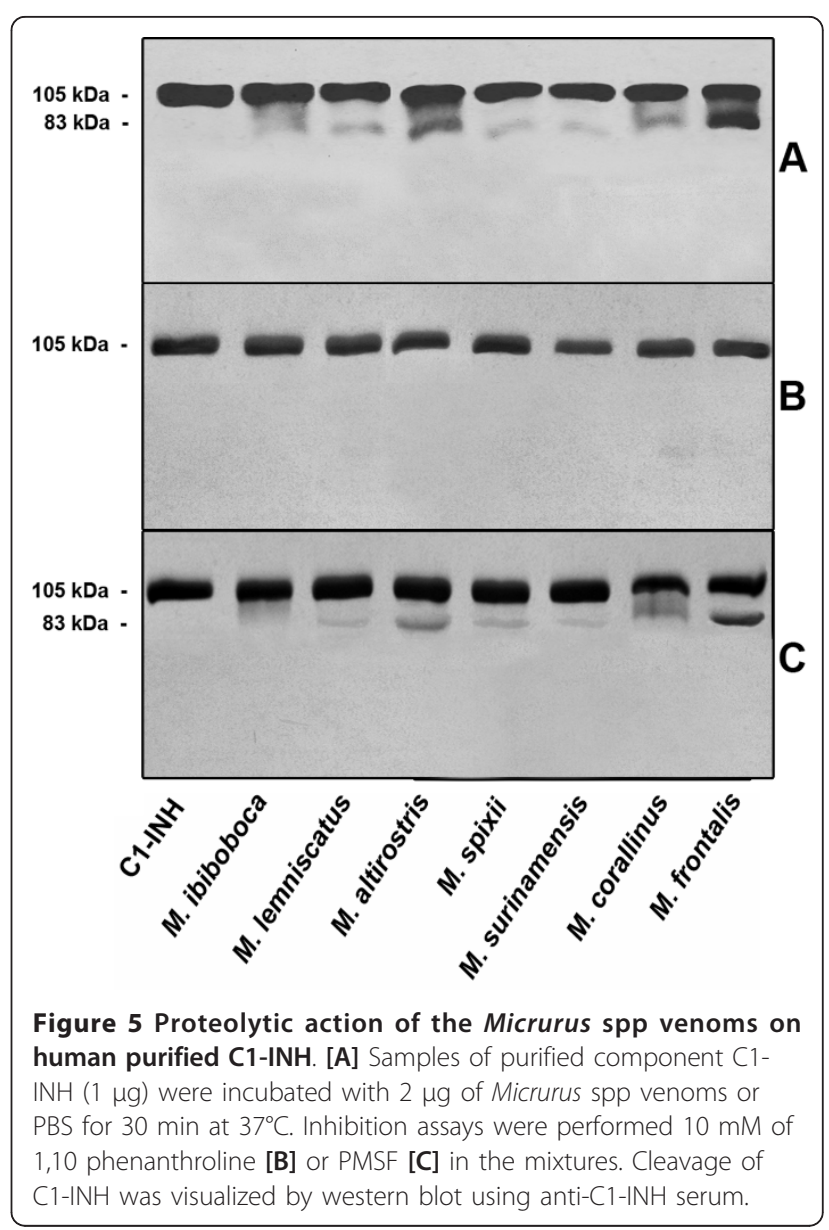

function is local acute inflammation at the site of bite. Some potentially pro-inflammatory factors such as proteolytic enzymes and activators of clotting, complement and kallikrein-kinin systems have been detected in Crotalidae and Viperidae venoms. The action of these factors on the corresponding host substrates may release endogenous mediators of inflammation [14,19-22].

The Elapidae venoms analyzed in the present study from Micrurus genus were also able to activate the complement system. M. ibiboboca and M. altirostris venoms affected classical and lectin pathways; M. spixii and M. frontalis venoms affected only the classical pathway and $M$. lemniscatus, $M$. surinamensis and $M$. corallinus venoms only the lectin pathway. These differences of Micrurus spp venoms action on the complement system may be due to interspecies variations in venom composition. Indeed, previous results of our group [6] showed that venoms from these Micrurus species differ in protein composition and toxic potential, probably associated with the geographical origin, habitat and diet of the snakes.

The majority of the Micrurus spp venoms induced the production of significant amounts of the three anaphylatoxins C3a, C4a and C5a. This suggests that these venoms 
are able to activate the complement system and that the observed reduction of the $\mathrm{CP}$ and LP complement activities, after treatment with the various venoms, was most likely caused by activation of the pathways, resulting in consumption of the C-components; however some inactivation/inhibition of $\mathrm{C}$-components may also have occurred.

The action of cobra venom factor (CVF) from the cobra Naja naja, as a complement activator and generator of anaphylatoxins, has been well described [14-16]. In the present study, the presence of CVF-like molecules was not detected in Micrurus spp venoms, using antibodies against $N$. naja CVF in western blot assays. These results may suggest that CVF components are not present in the Micrurus venoms here studied or that they do not share the same antigenic properties.

Analysis of a possible direct proteolytic action of Micrurus venoms on the complement molecules showed that, using purified C3, cleavage was induced by $M$. ibiboboca, M. lemniscatus, $M$. corallinus, $M$. frontalis but not by $M$. altirostris, $M$. spixii and $M$. surinamensis. C3 proteolytic activity in snake venoms from the Elapidae family has been previously reported by O'Keefe and collaborators [23] in Naja naja siamensis and by us in $N$. naja, N. nigricollis and M. ibiboboca [7], although no further characterization of the class of protease involved has been performed. In the present study, we have shown that metalloproteinases are the main proteases responsible for this event, since the specific inhibitor, 1,10 phenathroline, completely abrogated the C3 $\alpha$ chain hydrolysis induced by Micrurus venoms, thus, possibly contributing to the activation and inactivation of the complement activation pathways, as well as to the generation of the anaphylatoxins. On the other hand, no cleavage of $\mathrm{C} 4$ was observed, suggesting the presence of a specific $\mathrm{C} 3 \alpha$ chain cleaving metalloprotease in some Micrurus venoms.

The majority of the Micrurus spp venoms induced consumption of the classical and or the lectin pathways and we investigated the mechanism of this. The presence of antibodies as initiating factor was discarded, since the human serum samples used were devoid of Micrurus spp venoms specific antibodies (data not shown). Another possibility investigated was the failure of the complement regulatory mechanisms. $\mathrm{C} 1-\mathrm{INH}$ is the primary regulator of the activation of the classical and lectin pathways and also plays an important role in the regulation of coagulation and fibrinolysis. $\mathrm{C} 1-\mathrm{INH}$ inactivates $\mathrm{C} 1 \mathrm{r}$ and $\mathrm{C} 1 \mathrm{~s}$, the serine protease subcomponents of the first component of the classical pathway, and MASP 1 and 2 proteases of the lectin pathway; removal/inactivation of $\mathrm{C} 1$-INH leads to auto-activation of the CP and LP. We show here that all Micrurus venoms were able to cleave the C1-INH, generating a fragment of approximately $83 \mathrm{kDa}$, and this hydrolysis could be blocked by metalloproteinase inhibitors 1,10 phenanthroline. PMSF could partially abolish the cleavage of $\mathrm{C} 1-\mathrm{INH}$ induced by $M$. ibiboboca venom.

Cleavage of C1-INH by Crotalid, Viperid and Colubrid snake venoms has previously been reported by Kress et al. [24], who demonstrated that the inhibitor was converted into an active intermediate species of $89-\mathrm{kDa}$ and then a further cleavage resulted in formation of an $86-\mathrm{kDa}$ inactive inhibitor. We have recently demonstrated that venoms snakes from Bothrops genus were also able to cleave C1$\mathrm{INH}$, generating a main fragment of approximately $83 \mathrm{kDa}$ [17] and this fragmentation could be totally blocked by 1,10 phenanthroline. These data suggest that the different families of snakes may possess common factor(s) in their venoms allowing them to inactivate $\mathrm{C} 1-\mathrm{INH}$, which may lead to unrestricted activation of both coagulation and complement cascades and participate in the mediation of the toxic effects of the venoms.

\section{Conclusion}

Data presented here demonstrate that Micrurus venoms can activate the complement system, generating significant amounts of anaphylatoxins, which may assist, due to their vasodilatory effects, to enhance the spreading of other venom components.

\section{Methods}

\section{Chemicals and reagents}

Tween 20, bovine serum albumin (BSA), ortho-phenylenediamine (OPD), phenylmethylsulfonyl fluoride (PMSF), 1,10 phenanthroline, ethylene diamine tetracetic acid (EDTA), ethylene glycol bis-( $\beta$-aminoethyl ether)-N, $N, N$, N'-tetracetic acid (EGTA) and mannan were purchased from Sigma (St. Louis, Missouri, USA). Goat anti-rabbit (GAR) and rabbit anti-goat (RAG) IgG labelled with alkaline phosphatase (IgG-AP) or with horseradish peroxidase (IgG-HRPO), 5-bromo-4-chloro-3-indolyl-phosphate (BCIP) and nitroblue tetrazolium (NBT) were from Promega Corp. (Madison, Wisconsin, USA). Purified human C3 and C4, and goat IgG anti-human C4 were from Quidell Corporation (San Diego, CA, USA). Purified human C1-INH and rabbit IgG anti-C1-INH were from Calbiochem-Novabiochem Corp. (San Diego, CA, USA). Rabbit IgG anti-C3 was from Santa Cruz Biotechnology Inc. (Santa Cruz, CA, USA) and rabbit polyclonal serum against sheep erythrocytes was made in house. Polyclonal rabbit antiserum against Naja naja cobra venom factor (CVF) was a kind donation from Dr. Carl Wilhelm Vogel, Honolulu, Hawaii, USA.

\section{Venoms}

Venoms from Micrurus ibiboboca, M. lemniscatus, M. altirostris, M. spixii, M. surinamensis, M. corallinus, M. frontalis, Naja naja, Naja nigricollis, Naja mossambica 
and Naja melanoleuca were supplied by Herpetology Laboratory from Butantan Institute, SP, Brazil. Stock solutions were prepared in PBS $(10 \mathrm{mM}$ sodium phosphate containing $150 \mathrm{mM} \mathrm{NaCl}, \mathrm{pH} 7.2)$ at $1.0 \mathrm{mg} / \mathrm{mL}$. The permission to access venoms from Micrurus spp snakes (permission no. 01/2009) was provided by the Brazilian Institute of Environment and Renewable Natural Resources (IBAMA).

\section{Human serum and erythrocytes}

Human blood was obtained from healthy donors. Blood samples drawn to obtain sera were collected without anticoagulant and allowed to clot for $2 \mathrm{~h}$ at room temperature; the normal human serum (NHS) was stored at $-80^{\circ} \mathrm{C}$. Blood samples from sheep or rabbit, drawn to obtain erythrocytes for subsequent use as target cells, were collected in anticoagulant (Alsever's old solution: $114 \mathrm{mM}$ citrate, $27 \mathrm{mM}$ glucose, $72 \mathrm{mM} \mathrm{NaCl}, \mathrm{pH}$ 6.1). The human blood samples in this study were obtained from three healthy donors in our research group who clearly knew the purpose of this study, according to the Blood Donation Law of Brazil. This study was approved by the ethics committee of Butantan Institute.

\section{Treatment of the normal human serum with Micrurus spp venoms}

Samples of $50 \mu \mathrm{l}$ of normal human serum (NHS) were incubated with $50 \mu \mathrm{l}(50 \mu \mathrm{g})$ of Micrurus spp venoms or $\mathrm{PBS}$. The mixtures were incubated for $30 \mathrm{~min}$ at $37^{\circ} \mathrm{C}$ and tested for the residual complement activity in hemolytic assays (for classical and alternative pathways measurements) or by ELISA (for lectin pathway evaluation).

\section{Haemolytic complement activity}

For classical pathway analysis, sheep erythrocytes were washed in PBS and a $2 \%$ suspension was incubated with an equal volume of a 1:500 dilution in PBS of rabbit antisheep erythrocytes serum for $15 \mathrm{~min}$ at $37^{\circ} \mathrm{C}$. The antibody-sensitized sheep erythrocytes were washed and resuspended in veronal buffered saline $\left(\mathrm{VBS}^{++}: 3.7 \mathrm{mM}\right.$ Barbitone, $0.3 \mathrm{mM} \mathrm{CaCl}_{2}$ and $0.8 \mathrm{mM} \mathrm{MgCl}$, $145.5 \mathrm{mM}$ $\mathrm{NaCl}, \mathrm{pH} 7.2)$ at $2 \%$ final concentration. For alternative pathway analysis, rabbit erythrocytes were washed in PBS and resuspended in AP buffer ( $5 \mathrm{mM} \mathrm{Na}$-barbital, $10 \mathrm{mM}$ EGTA, $7 \mathrm{mM} \mathrm{MgCl} 2,150 \mathrm{mM} \mathrm{NaCl}, \mathrm{pH}$ 7.4) at $2 \%$ final concentration. The antibody-sensitized sheep erythrocytes or the rabbit erythrocytes $(50 \mu \mathrm{l})$ were added to each well, of a 96-well plate, and incubated, for $30 \mathrm{~min}$ at $37^{\circ} \mathrm{C}$, with venom- or PBS-treated NHS samples. For each sample to be tested doubling dilutions in $\mathrm{VBS}^{++}$were made $(150 \mu \mathrm{l} /$ well), including zero and $100 \%$ lysis wells. Unlysed cells were removed by centrifugation at $1300 \mathrm{rpm}$ at $4^{\circ} \mathrm{C}$ for 5 min. Fifty microlitres of each supernatant were transferred to new 96-well plates containing $200 \mu \mathrm{l}$ of water, and the absorbance measured at $414 \mathrm{~nm}$ as an index of hemolysis. Percentage of hemolysis for each well and the number of $\mathrm{CH}_{50}$ and $\mathrm{AP}_{50}$ for each serum were calculated by standard methods.

\section{Complement activation of the lectin pathway as determined by ELISA}

Microtitre plates were coated with $10 \mu \mathrm{g} /$ well of mannan, overnight at $4^{\circ} \mathrm{C}$, washed three times with $\mathrm{PBS} / 0.05 \%$ Tween 20, and blocked using 1\% BSA in PBS for $30 \mathrm{~min}$ at $37^{\circ} \mathrm{C}$. After washing, serial dilutions of NHS samples treated with PBS or Micrurus spp venoms were added. After $1 \mathrm{~h}$ of incubation at $37^{\circ} \mathrm{C}$, plates were washed with $\mathrm{BVB}^{++}\left(\mathrm{VBS}^{++}, \mathrm{pH} 7.2\right.$, containing $\left.0.1 \% \mathrm{BSA}\right)$ and incubated with anti-human $\mathrm{C} 4(1: 2.000)$ for $1 \mathrm{~h}$ at $37^{\circ} \mathrm{C}$. Plates were washed three times with $\mathrm{BVB}^{++} /$Tween $0.05 \%$ and incubated with the specific anti-IgG antibody conjugated with HRPO for $1 \mathrm{~h}$. Plates were washed and the reactions developed with OPD substrate according to the conditions established by the manufacturers (Sigma). The absorbances were recorded in an ELISA reader (Multiskan spectrophotometer EX, Labsystems, Finland) at $\lambda 492 \mathrm{~nm}$. For complement activity ELISA, normal human serum arbitrarily set at $1000 \mathrm{aU} / \mathrm{ml}$, was used to produce a calibration curve.

\section{Detection of Anaphylatoxins}

Samples of normal human serum $(50 \mu \mathrm{L})$ were incubated with PBS $(50 \mu \mathrm{L})$ or with Micrurus spp venoms $(50 \mu \mathrm{L}=$ $50 \mu \mathrm{g}$ ) for $30 \mathrm{~min}$ at $37^{\circ} \mathrm{C}$, and $\mathrm{C} 3 \mathrm{a} / \mathrm{C} 3 \mathrm{a}$ desArg, $\mathrm{C} 4 \mathrm{a} / \mathrm{C} 4 \mathrm{a}$ desArg, and $\mathrm{C} 5 \mathrm{a} / \mathrm{C} 5 \mathrm{a}$ desArg concentrations were measured using the Cytometric Bead Array (BD Biosciences Pharmingen, New Jersey, California, USA). Anaphylatoxin capture beads were incubated with the standards or with the test samples, washed, and then incubated with phycoerythrin-conjugated detection antibodies to form sandwich complexes. Two-color flow cytometric analysis was performed using a FACSCalibur flow cytometer (Becton Dickinson Immunocytometry Systems, Santa Clara, California, USA). Data were acquired and analyzed using Becton Dickinson Cytometric Bead Array CBA software. Anaphylatoxin concentrations were determined from the standard curves, plotting anaphylatoxin calibrator concentration versus FL-2 mean fluorescence intensity.

\section{Proteolytic activity of Micrurus spp venoms on the complement molecules}

Venoms samples $(1 \mu \mathrm{g})$ were incubated with the human purified components C3, C4 or C1-INH $(2 \mu \mathrm{g})$ in PBS for $30 \mathrm{~min}$ at $37^{\circ} \mathrm{C}$, and the cleavage was detected by western blotting. $20 \mathrm{mM}$ of PMSF or phenanthroline, inhibitors of serine- and metallo-proteases, respectively, 
were added to assess the nature of the cleaving enzyme in the venom.

\section{Electrophoresis and western blot}

Samples were solubilised in reducing or non-reducing sample buffers and separated in 10\% SDS-PAGE gels [25]. Gels were blotted onto nitrocellulose [26]. After transfer, the membranes were blocked with PBS containing 5\% BSA for $2 \mathrm{~h}$ and incubated with anti-C3 (1:5000), anti-C4 (1:1000), anti-C1-INH human (1:2000) or anti-CVF (1:1500) sera, for $1 \mathrm{~h}$ at room temperature. Immunoreactive proteins were detected using GAR/IgGAP or RAG/IgG-AP (1:7500) in PBS/1\% BSA for $1 \mathrm{~h}$ at room temperature. After washing 3 times, for $10 \mathrm{~min}$ with $\mathrm{PBS} / 0.05 \%$ Tween 20 , blots were developed using NBT/BCIP according to the manufacturer's instructions (Promega).

\section{Abbreviations used}

C: Complement system; CP: classical pathway; AP: alternative pathway; LP: Lectin pathway; C1-esterease Inhibitor: C1-INH; CVF: cobra venom factor; NHS: normal human serum.

\section{Acknowledgements}

This research was supported by grants from FAPESP, CNPq and INCTTOX.

\section{Author details}

${ }^{1}$ Immunochemistry Laboratory, Butantan Institute, Av. Vital Brazil, 1500, São Paulo, 05503-900, Brazil. ${ }^{2}$ Herpetology Laboratory, Butantan Institute, Av. Vital Brazil, 1500, São Paulo, 05503-900, Brazil. 'Department of Pharmacology, Oncology and Radiology, School of Medicine, Cardiff University, Cardiff CF14 $4 \mathrm{XN}, \mathrm{UK}$

\section{Authors' contributions}

All authors have read and approved the final manuscript.

Conceived and designed the experiments: GDT, GPQ, MFDF, DVT. Performed the experiments: GDT, GPQ. Analyzed the data: GDT, GPQ, CWVB, DVT. Contributed with reagents/materials: MFDF, DVT. Wrote the paper: GDT, CWVB, DVT.

Received: 3 November 2011 Accepted: 16 January 2012

Published: 16 January 2012

\section{References}

1. Vital Brazil O, Fontana MD, Pellegrini Filho A: Physiopathologie et therapeutique de l'envenomation experimentale causee par le venin de Micrurus frontalis. Mem Inst Butantan 1976, 40:221-240.

2. Barros ACS, Fernandes DP, Ferreira LCL, Santos MC: Local effects induce by venoms from five species of genus Micrurus sp (coral snakes). Toxicon 1994, 32:445-452.

3. Gutiérrez JM, Lomonte B, Portilla E, Cerdas L, Rojas E: Local effects induced by coral snake venoms: evidence of myonecrosis after experimental inoculation of venoms from five species. Toxicon 1983, 21:777-783.

4. Tan NH, Ponnudurai G: The biologial properties of venoms of some american coral snake (genus Micrurus). Comp Biochem Physiol 1992, 101B:471-474

5. Aird SD, Jorge da Silva N: Comparative enzymatic composition of Brazilian coral snake (Micrurus) venoms. Comp Biochem Physiol 1991, 99B:287-294.

6. Tanaka GD, Furtado MDFD, Portaro FCV, Sant'Anna OA, Tambourgi DV Diversity of Micrurus snake species related to their venom toxic effects and the prospective of antivenom neutralization. PLoS Negl Trop Dis 2010, 4:e622.

7. Tambourgi DV, Santos MC, Furtado MF, Freitas MC, Silva WD, Kipnis TL: Proinflammatory activities in elapid snake venoms. Br J Pharmacol 1994, 112:723-727.

8. Köhl J: Self, non-self, and danger: a complementary view. Adv Exp Med Biol 2006, 586:71-94.

9. Beinrohr L, Dobó J, Závodszky P, Gál P: C1, MBL-MASPs and C1-inhibitor: novel approaches for targeting complement-mediated inflammation. Trends Mol Med 2008, 14:511-521.

10. Unsworth DJ: Complement deficiency and disease. J Clin Pathol 2008, 61:1013-1017.

11. Sjöberg AP, Trouw LA, Blom AM: Complement activation and inhibition: a delicate balance. Trends Immunol 2009, 30:83-90.

12. Bowen B, Hawk JJ, Sibunka S, Hovick S, Weiler JM: A review of the reported defects in the human $\mathrm{C} 1$ esterase inhibitor gene producing hereditary angioedema including four new mutations. Clin Immunol 2001, 98:157-163.

13. Miwa T, Song WC: Membrane complement regulatory proteins: insight from animal studies and relevance to human diseases. Int Immunopharmacol 2001, 1:445-459.

14. Müller-Eberhard HJ, Fjellstrom KE: Isolation of the anti-complementary protein from cobra venom and its mode of action on C3. J Immunol 1971, 6:1666-1672.

15. Götze O, Müller-Eberhard HJ: The C3-activator system: an alternate pathway of complement-activation. J Exp Med 1971, 3:90-108.

16. Vogel CW, Müller-Eberhard HJ: The cobra venom factor-dependent C3 convertase of human complement. A kinetic and thermodynamic analysis of a protease acting on its natural high molecular weight substrate. J Biol Chem 1982, 14:8292-8299.

17. Pidde-Queiroz G, Furtado MDFD, Filgueiras CF, Pessoa LA, SpadaforaFerreira M, van den Berg CW, Tambourgi DV: Human complement activation and anaphylatoxins generation induced by snake venom toxins from Bothrops genus. Mol Immunol 2010, 47:2537-2544

18. Zeller EA: Enzymes of snake venoms and their biological significance. Adv Enzymol 1948, 8:459-495.

19. Rocha-e-Silva M, Beraldo WT, Rosenfeld G: Bradykinin, a hypotensive and smooth muscle stimulating factor released from plasma globulin by snake venoms and trypsin. Am J Physiol 1950, 156:261-273.

20. Stocker K, Barlow GH: The coagulant enzyme from Bothrops atrox venom (batroxobin). Methods Enzymol 1976, 45:214-223.

21. Geiger R, Kortmann H: Esterolytic and proteolytic activities of snake venoms and their inhibition by proteinase inhibitors. Toxicon 1977, 15:257-259.

22. Stocker $\mathrm{K}$, Fisher $\mathrm{H}$, Meier J: Thrombin-like snake venom proteinases. Toxicon 1982, 20:265-273.

23. O'Keefe MC, Caporale LH, Vogel CW: A novel cleavage product of human complement component C3 with structural and functional properties of cobra venom factor. J Biol Chem 1988, 263:12690-12697.

24. Kress LF, Catanese J, Hirayama T: Analysis of the effects of snake venom proteinases on the activity of human plasma C1 esterase inhibitor, alpha 1-antichymotrypsin and alpha 2-antiplasmin. Biochim Biophys Acta 1983, 15:113-120.

25. Laemmli UK: Clevage of structural proteins during the assembly of the head of bacteriophage $T_{4}$. Nature 1970, 227:680-685.

26. Towbin H, Staehelin T, Gordon J: Electrophoretic transfer of proteins from polyacrylamide gels to nitrocellulose sheets: procedure and some applications. Biotechnology 1979, 24:145-149.

doi:10.1186/1471-2172-13-4

Cite this article as: Tanaka et al:: Micrurus snake venoms activate human complement system and generate anaphylatoxins. BMC Immunology 2012 13:4. 